\title{
(6) OPEN ACCESS \\ Impact of ethnicity upon cardiovascular adaptation in competitive athletes: relevance to preparticipation screening
}

\author{
Michael Papadakis, ${ }^{1}$ Mathew G Wilson, ${ }^{2}$ Saqib Ghani, ${ }^{1}$ Gaelle Kervio, ${ }^{3}$ Francois Carre, ${ }^{3}$ \\ Sanjay Sharma ${ }^{1}$
}

'Department of Cardiology, St George's University of London, London, UK ${ }^{2}$ Department of Sports Medicine, Aspetar, Oatar Orthopaedic and Sports Medicine Hospital, Doha, Oata ${ }^{3}$ French Institute of Health and Medical Research (INSERM) Rennes, F-35000, France

\section{Correspondence to} Professor Sanjay Sharma, Department of Cardiology, St George's University of London, Cranmer Terrace, London SW17 ORE, UK; ssharma21@hotmail. com

Received 28 February 2012 Accepted 4 July 2012 Published Online First 26 July 2012

\begin{abstract}
Regular participation in intensive physical exercise is associated with several structural and electrophysiological cardiac adaptations that enhance diastolic filling and facilitate a sustained increase in the cardiac output that is fundamental to athletic excellence. Such cardiac adaptations are collectively referred to as the 'Athlete's Heart' and are frequently reflected on the 12-lead ECG and imaging studies. Thorough knowledge relating to exercise-associated cardiovascular adaptation is imperative for the purposes of differentiating physiological adaptation from cardiac pathology, since an erroneous diagnosis of cardiac disease has potentially serious consequences for the athlete's physical, psychological, social and financial well-being. The majority of studies investigating the cardiovascular adaptation to exercise are based on cohorts of Caucasian athletes. However, there is mounting evidence that ethnicity is an important determinant of the objective manifestations of cardiovascular adaptation to exercise. The most pronounced paradigm of ethnically distinct cardiovascular adaptation to exercise stems from athletes of African/ Afro-Caribbean descent, who exhibit a significantly higher prevalence of repolarisation anomalies and left ventricular hypertrophy, compared to Caucasian athletes; the differentiation between athlete's heart and hypertrophic cardiomyopathy is particularly challenging in this ethnic group. The extrapolation of ECG and echocardiographic criteria used to diagnose potentially serious cardiac disorders in Caucasian athletes to the African/AfroCaribbean athlete population would result in an unacceptable number of unnecessary investigations and increased risk of false disqualification from competitive sport. Accurate interpretation of the athlete's ECG and echocardiogram is crucial, particularly when one considers the continuous expansion of preparticipation screening programmes. This review attempts to highlight ethnically determined differences in cardiovascular adaptation to exercise and provides a practical guide for the interpretation of baseline investigations in athletes of diverse ethnic backgrounds.
\end{abstract}

\section{INTRODUCTION}

Competitive sports place a high premium on athletic excellence and participating athletes frequently subject themselves to arduous training regimes, which often exceed their innate physical limits. A prerequisite for such physical achievements is an increase in the cardiac output during exertion, which may need to be sustained for prolonged periods. ${ }^{1}$ The term 'Athlete's Heart' refers to a constellation of cardiovascular adaptations affecting the structure, electrical conduction and function of the heart, which facilitate substantial increases in cardiac output during exercise. The objective markers of such physiological adaptations are frequently reflected on the 12-lead ECG as well as imaging and functional studies.

The vast majority of athletes exhibit relatively mild structural and electrical changes, which are considered to be within conventionally defined normal limits. A small proportion of athletes develop pronounced changes, which overlap with phenotypic expressions of cardiac diseases implicated in exercise-associated sudden cardiac death (SCD). In such circumstances, the differentiation between physiological adaptation and cardiac pathology is challenging but an erroneous diagnosis has the potential for grave consequences.

It has been well established that the quantitative and qualitative manifestations of athlete's heart are influenced by several factors including the size, age, sex and sporting discipline of the athlete (figure 1). ${ }^{1}$ The past few decades have observed an exponential increase in the number of athletes of African, Arabic and South American descent that excel at highly competitive level, prompting researchers to investigate the effect of exercise in those ethnicities. The results categorically indicate that ethnicity is an important determinant of cardiovascular adaptation. Until recently, however, most studies delineating the clinical manifestations of the athlete's heart were based on investigations performed solely on Caucasian athletes and criteria derived from such studies continue to form the 'gold-standard' for differentiating physiological adaptation from clinical expressions of potentially fatal cardiac disorders. $^{2}{ }^{3}$ In the light of new knowledge relating to the impact of ethnicity on the athlete's heart, it would appear unsatisfactory to apply ECG or imaging criteria denoting abnormal tests in Caucasian athletes to athletes of other ethnicities.

This article provides a comprehensive review of available data in an attempt to highlight ethnically determined differences in cardiovascular adaptation to exercise and offers a practical guide for the interpretation of baseline investigations in athletes of different ethnic backgrounds.

\section{METHODOLOGY}

We searched PubMed and Medline from 1950 onwards using the search terms 'athletes', 'ethnicity', 'black athlete', 'Asian athlete', 'cardiovascular adaptation', 'electrocardiogram', 'preparticipation 


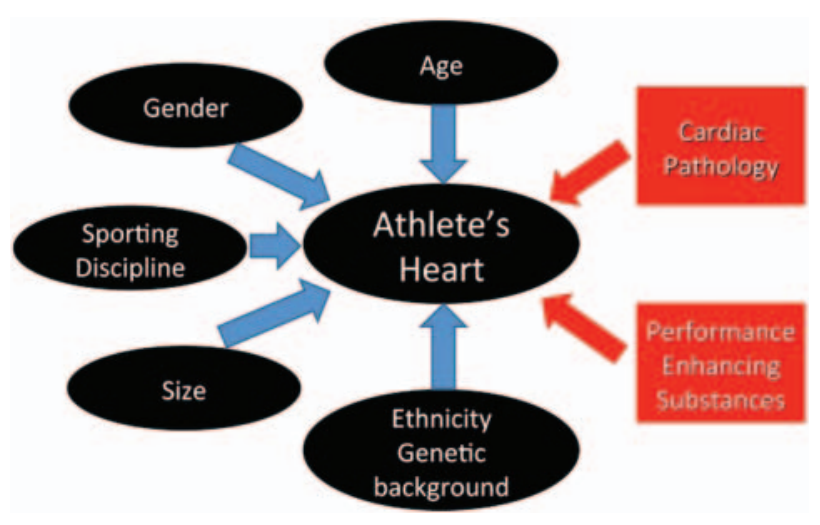

Figure 1 Schematic representation of demographic and pathological factors, which may influence the cardiovascular adaptation to exercise and as a consequence its phenotypic manifestation on the 12-lead ECG and imaging studies. This figure is only reproduced in colour in the online version.

screening' and 'sudden death'. We scrutinised and selected the highest quality articles, comprising original papers, reviews, recommendations and consensus reports. All studies are population based, prospective or retrospective observational reports.

\section{IMPACT OF ETHNICITY UPON CARDIOVASCACULAR ADAPTATION TO EXERCISE}

\section{Cardiovascular adaptation in Caucasian athletes}

The concept that the cardiovascular system of trained athletes differs from sedentary individuals is well established. ${ }^{4}$ Cardiovascular adaptation depends on the type of conditioning, namely endurance or strength training. Most athletic disciplines combine both types of training and the precise manifestations of cardiac adaptation exhibited during objective assessment are dependent on complex haemodynamic and metabolic interactions at multiple levels. ${ }^{5}$

\section{Structural adaptations}

Training induces some evidence of cardiac remodelling in approximately $50 \%$ of athletes. ${ }^{5}$ Most data are derived from cross-sectional echocardiographic studies in highly trained athletes and demonstrate dilatation of all cardiac chambers and an increase in the left ventricle (LV) wall thickness (LVWT).

Makan et al demonstrated a significant increase in the absolute LV dimensions in highly trained adolescent (14-16 years) athletes compared to sedentary controls matched for age, sex and size. The LV cavity ranged from 52 to $60 \mathrm{~mm}$ and was $>54 \mathrm{~mm}$ in $18 \%$ athletes. ${ }^{6}$ Similarly, a study by Pelliccia et al ${ }^{7}$ in Olympian Italian athletes revealed that almost half of the cohort exhibited an LV cavity size of $>55 \mathrm{~mm}$ and $14 \%$ exhibited extreme LV cavity dilatation (>60 mm), which resemble those in patients with dilated cardiomyopathy. None of the athletes with dilated LV cavities demonstrated any evidence of LV systolic dysfunction, and in the majority of athletes detraining resulted in significant reduction of the LV cavity dimensions. ${ }^{8}$ The same group demonstrated left atrial (LA) dilatation $(\geq 40 \mathrm{~mm}$ ) in $20 \%$ of elite athletes, which largely correlated with LV cavity enlargement and volume overload, but was not associated with increased incidence of supraventricular arrhythmias. ${ }^{9}$ Limited data are available regarding the effect of athletic training on the right cardiac chambers, primarily due to the echocardiographic limitations of imaging the right heart. Emerging data from cardiac MRI suggest a balanced structural adaptation between the left and right chambers. ${ }^{10}$ Finally, the aortic root also dilates in highly trained athletes but usually does not exceed $40 \mathrm{~mm}^{11}$

Left ventricular hypertrophy (LVH) may be eccentric as a result of the dilated cavity in endurance athletes or concentric in both endurance and strength-trained athletes. Within a cohort of 947 athletes however, only $1.7 \%$ of male and none of the female participants developed an LVWT of $>12 \mathrm{~mm} .{ }^{12}$ These findings have been replicated in numerous other studies and the arbitrary cut-off of $\geq 13 \mathrm{~mm}$ is used as an indication for further evaluation to exclude a morphologically mild hypertrophic cardiomyopathy (HCM) phenotype. ${ }^{13}$

\section{2-lead ECG}

In the majority of athletic individuals the 12-lead ECG exhibits alterations reflecting autonomic balance adaptations and increased cardiac chamber size (figure $2 \mathrm{~A}$ ), which are considered to be benign and training related (figure 3 ). A small proportion of athletes, however (4-7\%), exhibit ECG anomalies such as deep $\mathrm{T}$-wave inversions and pathological $\mathrm{Q}$-waves, which are frequently encountered in cardiomyopathies and require further comprehensive evaluation (figure 3). ${ }^{14-17}$ Results from a longitudinal study by the Pelliccia group suggest that although the bulk of such distinct ECG anomalies may represent extreme expressions of the athlete's heart, a significant minority of athletes with deep $\mathrm{T}$-wave inversions may subsequently develop overt cardiomyopathies associated with fatal arrhythmias. ${ }^{18}$

\section{Cardiovascular adaptation in African/Afro-Caribbean athletes}

The effect of African/Afro-Caribbean ethnicity on the cardiovascular system of both athletic and non-athletic individuals was documented in small, observational studies, as early as the 1950s, which described marked repolarisation changes including ST-segment elevation and deep T-wave inversions in African/ Afro-Caribbean individuals. ${ }^{19} 20$ In addition, echocardiographic studies in hypertensive subjects have shown a significantly greater LV mass index in African/Afro-Caribbean individuals compared with Caucasian individuals of similar age, gender and blood pressure readings. ${ }^{21}$ It is only over the past decade, however that the cardiovascular effects of exercise in African/ Afro-Caribbean athletes have been systematically studied, driven by an upsurge in the number of African/Afro-Caribbean athletes competing at elite level in Europe and the USA.

\section{Structural adaptations}

Studies by Papadakis et al and Basavarajaiah et al in highly trained, male British and French athletes revealed that African/ Afro-Caribbean athletes exhibit an LV cavity size of similar magnitude to white athletes $(52.6 \pm 4.4$ vs $52.6 \pm 4.3 \mathrm{~mm}$, $\mathrm{p}>0.05)$ but demonstrate slightly larger LA (35.4 \pm 4.5 vs 34.7 $\pm 4.7 \mathrm{~mm}, \quad \mathrm{p}=0.002)$ and aortic root $(30.2 \pm 3.3$ vs 29.5 $\pm 3.3 \mathrm{~mm}, \mathrm{p}<0.001$ ) diameters. ${ }^{22} 23$

However, the most striking difference between the two ethnic groups was the greater LV wall thickness measurement in African/Afro-Caribbean athletes, with $12.4 \%$ of the African/ Afro-Caribbean athletes exhibiting LVH (defined as an LV wall thickness $>12 \mathrm{~mm}$ ). In agreement with studies in Italian athletes, only $1.6 \%$ of Caucasian athletes exceeded an LV wall thickness $>12 \mathrm{~mm}$. None of the athletes exhibited a wall thickness $>16 \mathrm{~mm}$ (figure 4). All athletes with LVH revealed a normal or increased LV cavity size and normal diastolic indices, indicating that the LVH was reflective of intense training rather than a mild form of $\mathrm{HCM}^{23}$ Rawlins et al ${ }^{24}$ observed similar results in a large cohort of nationally ranked female athletes. Just over $3 \%$ of the African/Afro-Caribbean female athletes demonstrated a 


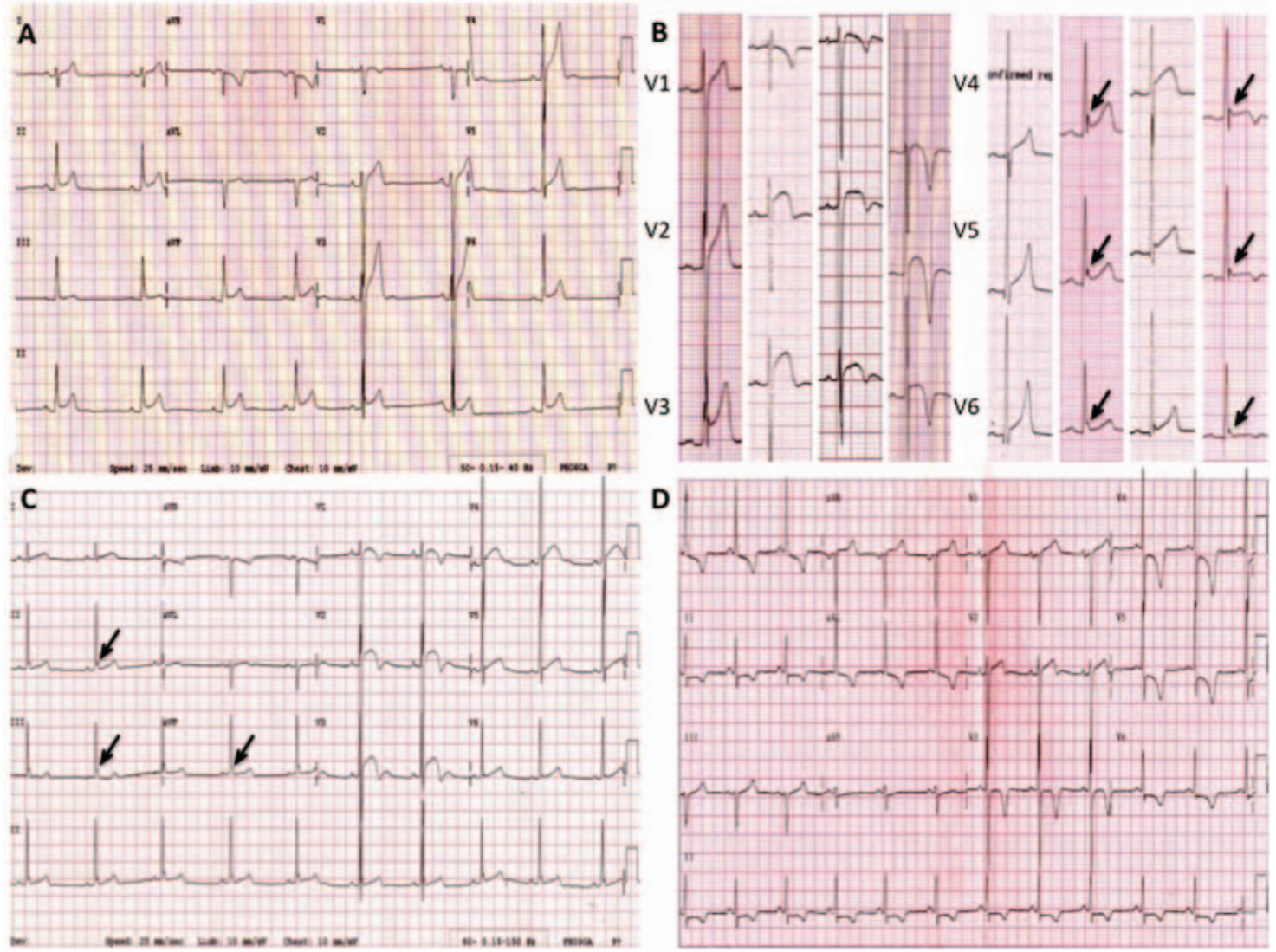

Figure 2 ECG examples demonstrating common repolarisation changes in Caucasian and African/Afro-Caribbean individuals. (A) Caucasian cyclist with typical benign (training-related) ECG changes; sinus bradycardia, large ORS complexes, ST-segment elevation and tall, tented T-waves. (B) Examples of common ST-segment morphologies in leads V1-V3 and V4-V6 in African/Afro-Caribbean athletes. Note the early repolarisation patterns in the lateral leads (arrows). (C) African/Afro-Caribbean long-distance runner with convex ST-segment elevation and deep T-wave inversions in leads V1-V3 and the early repolarisation pattern in the inferior leads (arrows). (D) African/Afro-Caribbean hypertrophic cardiomyopathy patient with ST-segment depression and deep T-wave inversions in the lateral leads. This figure is only reproduced in colour in the online version.

maximal LVWT of $>11 \mathrm{~mm}$ as opposed to none of the Caucasian athletes. Finally, a study by Di Paolo et a ${ }^{25}$ in adolescent (14-18 years) football players revealed that African athletes demonstrated greater mean LV wall thickness than Italian athletes of similar age $(9.7 \pm 1.3$ vs $9.2 \pm 1.0 \mathrm{~mm}, p=0.001)$, with

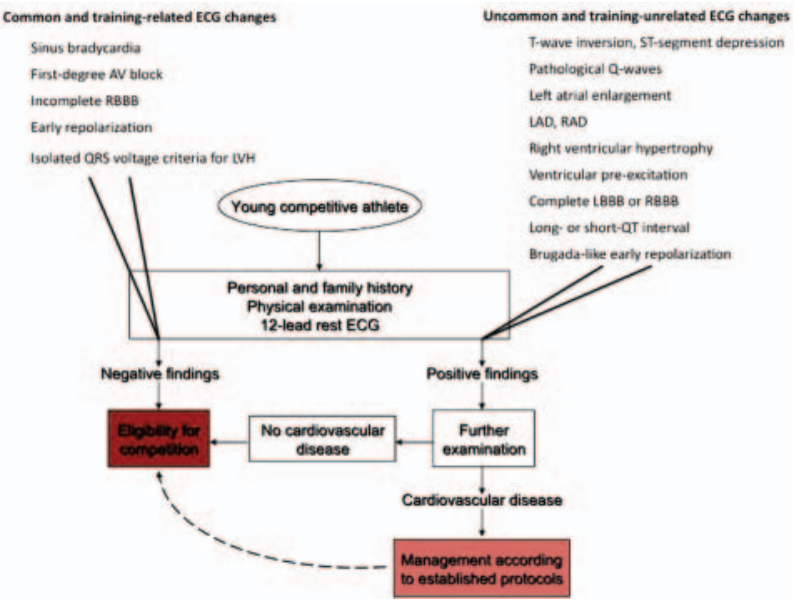

Figure 3 Diagrammatic representation of the preparticipation screening protocol for competitive athletes with associated common and training-related and uncommon and training-unrelated ECG changes. This figure is only reproduced in colour in the online version.
$2.6 \%$ of the African players exhibiting LVH compared to none of the Caucasian players. Unpublished data by our group examining cardiac adaptation in 1233 adolescent athletes from a variety of sporting disciplines, revealed that an LV wall thickness $>12 \mathrm{~mm}$ was present in an even higher proportion (7\%) of adolescent

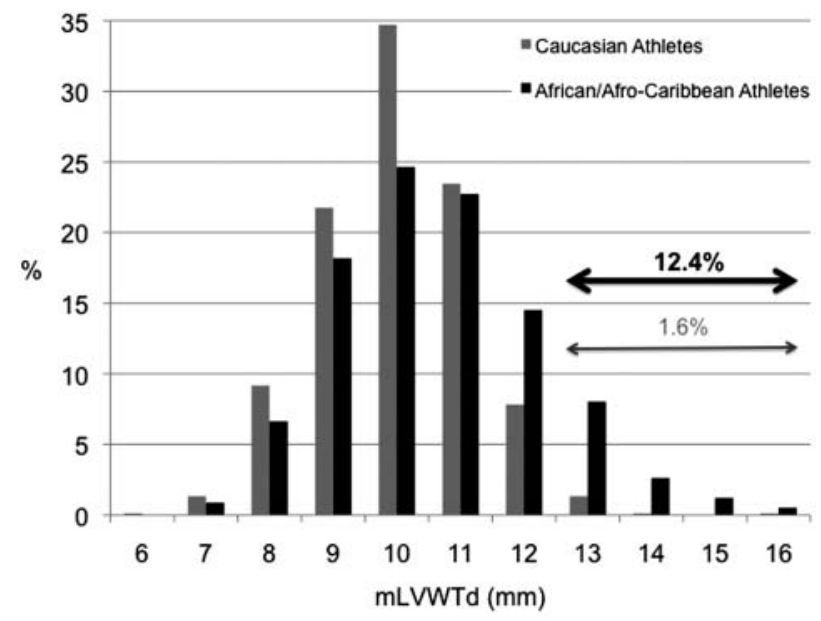

Figure 4 Histogram demonstrating the distribution of maximal left ventricular wall thickness at end-diastole (mLVWTd) as percentage $(\%)$ of the total African/Afro-Caribbean athlete (black bars) and Caucasian athlete (grey bars) cohort. $^{27}$ 
African/Afro-Caribbean athletes compared to only $0.6 \%$ of Caucasian athletes. Most importantly however, our study showed that even in the very young ( $<16$ years of age) athletes, $5.5 \%$ of adolescent African/Afro-Caribbean athletes but none of the adolescent Caucasian athletes exhibited LVH, with African/ Afro-Caribbean athletes as young as 14 years old recording an LVWT of up to $15 \mathrm{~mm}$.

\section{2-lead ECG}

According to previous studies in American football players, African/Afro-Caribbean athletes exhibit a greater prevalence of repolarisation changes compared to Caucasian counterparts. ${ }^{26} 27$ However, it was not until recently that a study by Papadakis et al attempted to quantify the prevalence, distribution, pattern and most importantly, the significance of repolarisation changes in African/Afro-Caribbean athletes. The authors compared ECGs of 904 elite male African/Afro-Caribbean athletes with those of 1819 Caucasian athletes, 119 African/AfroCaribbean sedentary individuals and 52 African/Afro-Caribbean patients with HCM. Patients with HCM were chosen for comparison since in clinical practice the presence of LVH and co-existent repolarisation anomalies frequently raises the diagnostic dilemma of physiological LVH versus HCM. ${ }^{23}$

T-wave inversions were present in $23 \%$ of African/ Afro-Caribbean athletes compared to only $3.7 \%$ of Caucasian athletes. T-wave inversions in African/Afro-Caribbean athletes were predominantly $(12.7 \%)$ confined to contiguous anterior leads (V1-V4) and only $4.1 \%$ of African/Afro-Caribbean athletes exhibited $\mathrm{T}$-wave inversions in the lateral leads. In contrast, both African/Afro-Caribbean controls and African/Afro-Caribbean HCM patients exhibited lower prevalence of $\mathrm{T}$-wave inversions in leads V1-V4 (4.2\% and 3.8\%, respectively) with most T-wave inversions in HCM patients (76.9\%) involving the lateral leads (figure 5). During subsequent comprehensive evaluation and follow-up (69.7 \pm 29.6 months) of a significant proportion of athletes, one African/Afro-Caribbean athlete survived a cardiac arrest and two athletes (one African/Afro-Caribbean athlete, one Caucasian athlete) were diagnosed with HCM. All three athletes exhibited T-wave inversions in the lateral leads. Based on these results the authors concluded that $\mathrm{T}$-wave inversions in leads V1-V4, commonly associated with convex ST-segment elevation in African/Afro-Caribbean athletes, are likely to represent an ethnic variant of 'athlete's heart'. Conversely, T-wave inversions in the lateral leads may represent the initial expression of HCM and merit further cardiovascular evaluation and regular follow-up. Almost $6 \%$ of African/Afro-Caribbean athletes exhibited $\mathrm{T}$-wave inversion in at least two inferior leads. The prevalence of $\mathrm{T}$-wave inversion in the inferior leads was not dissimilar to sedentary African/Afro-Caribbean individuals and African/ Afro-Caribbean patients with HCM, but the precise significance of this pattern of repolarisation has not been clarified.

Although ST-segment elevation was more prevalent in African/Afro-Caribbean athletes compared to Caucasian athletes (63.2\% vs $26.5 \%$, $\mathrm{p}<0.001)$, it was also highly prevalent $(65.5 \%)$ in African/Afro-Caribbean sedentary individuals, suggesting an ethnicity-related effect. More detailed inspection of the morphology of the ST-segments revealed that although the concave/ saddle-shaped patterns simulating acute pericarditis were common in both groups, convex ST-segment elevation in leads V1-V4 simulating acute anterior myocardial infarction or the Brugada phenotype was sixfold more common in the athletes, indicating a physiological response to training (figure $2 \mathrm{~B}, \mathrm{C}$ ).

Detailed review of the aforementioned studies suggests that ethnic differences of ECG phenotypes extend beyond

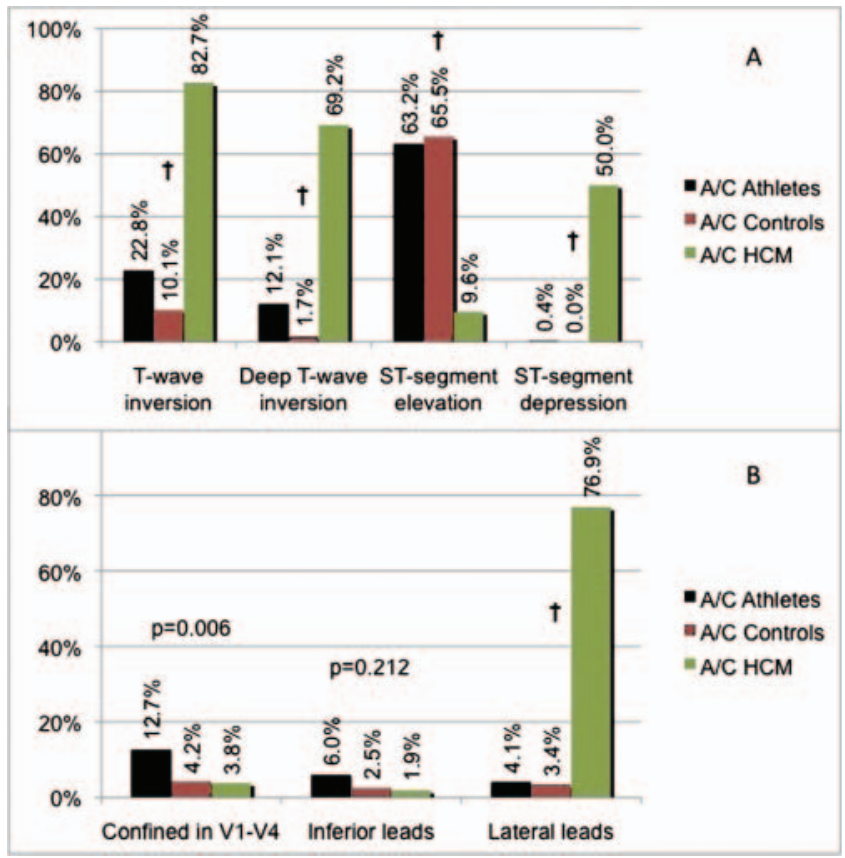

Figure 5 (A) Histogram demonstrating the prevalence of repolarisation changes as percentage (\%) of the total cohort in African/Afro-Caribbean $(\mathrm{A} / \mathrm{C})$ athletes, African/Afro-Caribbean $(\mathrm{A} / \mathrm{C})$ controls and African/ Afro-Caribbean $(A / C)$ hypertrophic cardiomyopathy patients (HCM) groups. (B) Histogram demonstrating the distribution of T-wave inversions as percentage (\%) of the total cohort in the three groups. ${ }^{27}$ $t p<0.001$ when comparing the three groups. This figure is only reproduced in colour in the online version.

repolarisation changes. In the study by Papadakis et al ${ }^{23}$ athletes of African/Afro-Caribbean ethnicity exhibited a higher prevalence of ECG voltage criteria for right ventricular hypertrophy $(13.3 \%$ vs $2.6 \%, \mathrm{p}<0.001)$ as well as right $(6.35 \%$ vs $0.3 \%$, $\mathrm{p}<0.001)$ and left $(8.6 \%$ vs $2.8 \%, \mathrm{p}<0.001)$ atrial enlargement.

Emerging evidence suggests that the early repolarisation (ER) pattern in the inferior leads of the 12-lead ECG may be associated with increased risk of SCD. ${ }^{28-30}$ Studies in athletes however indicate a high prevalence of the ER pattern (figure $2 \mathrm{~B}$ ), with highly variable estimates ranging from $20 \%$ to $90 \% .{ }^{31} 32$ In a longitudinal study of 148 athletes, Noseworthy et al demonstrated a correlation between ER and exercise. The prevalence of ER increased from $37.2 \%$ pretraining to $52.7 \%$ following the post-training period $(p=0.003)$. A significant increase with training was also noted for the inferior ER subtype (4.1\% pretraining vs $8.1 \%$ posttraining, $p=0.031$ ). The same study revealed a strong correlation between ER and African/Afro-Caribbean ethnicity (OR 5.84; $95 \%$ CI, 3.54 to $9.61 ; p<0.001)$. ER was observed in $61 \%$ of all African/Afro-Caribbean athletes and $18 \%$ exhibited ER in the inferior leads. During subsequent short-term follow-up (21 \pm 13 months) none of the athletes exhibited any significant clinical events. ${ }^{32}$ Unpublished data from our group confirm a high prevalence of the ER pattern in athletic individuals with a strong racial predisposition. Almost $40 \%$ of our African/Afro-Caribbean athletes' cohort exhibit ER, commonly in the inferior leads (figure 2C), compared to $20 \%$ of Caucasian athletes.

\section{Cardiovascular adaptation in West Asian (Arabic and Middle Eastern) athletes}

Structural adaptations

Limited echocardiographic data exist in elite West Asian athletes. An early study of 50 Iranian athletes demonstrated 
qualitatively similar changes in cardiac dimensions to Caucasian athletes; however, the study was too small to allow comment on physiological upper limits. ${ }^{33}$ Imaging data in large cohorts of West Asian athletes, who exhibit significantly smaller body surface area compared to their Caucasian counterparts, are required to ascertain whether the absolute values of upper physiological limits derived in Caucasian athletes can be applied to this ethnicity.

\section{2-lead ECG}

Until recently, there were no data relating to the ECG manifestations of cardiovascular adaptation in athletes of Arabic descent (Middle-Eastern and Gulf origin). In 2011, Wilson et al reported on the 12-lead ECG of 800 professional Arabic male athletes from seven Gulf States (Qatar, Bahrain, Oman, UAE, Kuwait, Yemen and Saudi-Arabia) and six Middle-Eastern countries (Egypt, Jordan, Palestine, Syria, Iraq and Lebanon) and compared them with 135 sedentary Arabic male controls as well as 120 Caucasian athletes. ${ }^{34}$ All participants underwent preparticipation screening. The ECG interpretation was based on the 2010 recommendations set by the European Society of Cardiology (ESC) (figure 3).

The authors observed that the prevalence of common and training-related ECG modifications were more prevalent in Caucasian athletes compared to Arabic athletes (98\% vs $91 \%$, $\mathrm{p}=0.008)$. On the contrary, uncommon and training unrelated ECG modifications were present in a similar proportion of both ethnic groups $(5.8 \%$ white athletes vs $7.9 \%$ Arabic athletes, $p>0.05)$. Of interest, in a small number of Arabic athletes the authors observed an elevated ST-segment with upward convexity, followed by T-wave inversion in leads V1-V4, commonly described in African/Afro-Caribbean athletes. In a similar manner to previous reports in Caucasian individuals, Arabic athletes demonstrated a significantly greater rate of uncommon ECG changes than Arabic controls (7.9\% vs. $0 \%$, p<0.05). ${ }^{34}$

\section{Cardiovascular adaptation in East Asian and South Asian athletes}

Limited data are available on the effects of cardiovascular adaptation in East Asian and South Asian athletes. The majority of data are derived from Chinese and Japanese athletes, but are limited by the small sample size. Moreover, due to cultural and social circumstances, there are no data on female athletes.

\section{Structural adaptations}

A study by Sun et al in elite Chinese athletes from a variety of sporting disciplines demonstrated that Chinese athletes exhibit LV cavity size dilatation and associated LVH of similar magnitude to Caucasian athletes. ${ }^{35}$ Of the 165 male athletes, $11.5 \%$ exhibited a LV cavity size $>60 \mathrm{~mm}$ with a maximum diameter of $65 \mathrm{~mm}$ and only $3(1.8 \%)$ athletes exhibited a wall thickness of $>12 \mathrm{~mm}$.

Nagashima et $a^{36}$ studied 291 Japanese male ultramarathon runners. A higher degree of chamber enlargement to those observed in Caucasian athletes was demonstrated with a mean LV cavity size of $61.8 \mathrm{~mm}$ (range $42-75 \mathrm{~mm}$ ). One-third of the athletes had an LV cavity diameter of more than $70 \mathrm{~mm}$. However, these results should be interpreted with caution given that the authors also reported LV wall thicknesses of up to $19 \mathrm{~mm}$, which would be highly suspicious of underlying cardiomyopathy, and none of the athletes were investigated comprehensively to exclude cardiac pathology. Although ethnicity might have been a determining factor, the older age (range 20-73 years) of the athletes and the intensity of training may also have influenced the results. A recent study by Kervio et al on 68 Japanese soccer players confirmed a higher LV enddiastolic diameter in Japanese compared to African/AfroCaribbean and Caucasian soccer players (55.2 \pm 3.3 vs $52.2 \pm$ 3.8 vs $53.9 \pm 3.7 \mathrm{~mm}$, respectively, $\mathrm{p}<0.01)$, with an important subset $(>4 \%)$ exhibiting markedly enlarged cavity (>60 mm), in the presence of normal systolic/diastolic function and no segmental abnormalities. ${ }^{37}$

\section{2-lead ECG}

The only available, large ECG study in East Asian and South Asian physically active individuals is a study in young, male recruits of the Singapore armed forces who underwent preparticipation cardiovascular screening utilising the 12-lead ECG. ${ }^{38}$ Out of 18476 participants (aged 16-27 years), 7\% exhibited an ECG abnormality requiring further evaluation. The prevalence of ECG abnormalities was higher in Chinese (7\%) compared to South Asian (5.7\%) recruits. The overall prevalence of repolarisation abnormalities such as $\mathrm{T}$-wave inversions and ST-segment depression was $1.3 \%$.

\section{CLINICAL IMPLICATIONS OF ETHNICALLY DETERMINED CARDIOVASCULAR ADAPTATION TO EXERCISE}

The American Heart Association (AHA) and the section of Sports Cardiology of the ESC advocate systematic preparticipation cardiovascular screening of competitive athletes aged 12-35 years old in an effort to identify athletes at risk of exercise-related cardiovascular events and SCD at an early stage. $^{39} 40$ The AHA recommends screening using a health questionnaire and physical examination. This strategy seems cheap and easy to carry out but has limited value as $80 \%$ of athletes who die as a result of SCD are asymptomatic and physical examination identifies few implicated disorders. ${ }^{41}$ The ESC recommendations are based on the Italian protocol, which utilises the 12-lead ECG. Athletes with ECG manifestations considered to represent uncommon or training-unrelated anomalies are subjected to a comprehensive clinical evaluation which may include transthoracic echocardiography or more advanced imaging studies, exercise testing, heart rhythm monitor recordings, evaluation of family relatives, genetic testing and even detraining and temporary disqualification from competition (figure 3).

Preparticipation screening is becoming a global phenomenon, involving athletes of diverse ethnic groups. ${ }^{42}$ Main sporting bodies including FIFA, UEFA and the International Olympic Committee have endorsed the European recommendations, despite limited understanding of the impact of ethnicity upon the 12-lead ECG and cardiac structure. ${ }^{3}$ Emerging studies suggest that ethnicity is an important determinant of cardiovascular adaptation to exercise, which should always be considered during assessment of an athlete. The authors offer a practical approach to screening athletes of non-Caucasian ethnicity based on adaptation of the current criteria to each ethnic group.

\section{Screening of African/Afro-Caribbean athletes}

Anterior T-wave inversions confined to leads V1-V4, especially when preceded by convex ST-segment elevation, are likely to represent an ethnically determined, physiological response to exercise. $^{23-25}$ In the absence of cardiac symptoms or a family history of SCD or cardiomyopathy the athlete may continue to compete without the need for further investigation. The authors concede however that the presence of physiological anterior T-wave inversions in African/Afro-Caribbean athletes 
may pose a significant challenge in identifying a small number of individuals who exhibit the ARVC phenotype, particularly in cases where T-wave inversions are not preceded by marked ST-segment elevation. In the absence of long-term follow-up data and lack of studies delineating the ECG morphology of African/Afro-Caribbean athletes with cardiomyopathy, individual physicians may opt to perform imaging studies.

The transthoracic echocardiogram of African/Afro-Caribbean athletes should be interpreted with caution, particularly in the context of coexistent repolarisation anomalies. Athletes of African/Afro-Caribbean origin are more likely to exhibit LVH compared to Caucasian counterparts with wall thicknesses of up to $16 \mathrm{~mm}$, which pose significant challenges in differentiating physiological LVH from HCM. This conundrum is of particular significance since exercise-related SCD secondary to HCM in the USA is reportedly higher in athletes of African/ Afro-Caribbean descent. ${ }^{43}$ A hypertrophic response to exercise may also be evident in athletes as young as 14 years old. With regard to gender differences African/Afro-Caribbean female athletes do not appear to exceed a LV wall thickness of more than $13 \mathrm{~mm}$. Based on available studies, it would be reasonable to infer that an absolute maximal LVWT of $16 \mathrm{~mm}$ and an absolute maximal LVWT of $13 \mathrm{~mm}$ probably represent the physiological upper limit of $\mathrm{LVH}$ in an asymptomatic male and female African/Afro-Caribbean athletes, respectively, outside the context of a family history of HCM.

\section{Screening of West Asian athletes}

The study by Wilson et al ${ }^{34}$ is the only large-scale study available in West Asian competitive athletes. The results suggests that West Asian athletes do not exhibit a higher prevalence of ECG changes compared to Caucasian athletes and as such when interpreting the ECG of West Asian competitive athletes the current ESC criteria could be adopted.

\section{Screening of East Asian and South Asian athletes}

The only available data relating to the effect of cardiovascular adaptation to exercise on the ECG of East Asian and South Asian athletes are based on a study in a large cohort of military recruits. ${ }^{38}$ The results suggest that East Asian and South Asian individuals exhibit a similar prevalence of ECG changes compared to Caucasian athletes. While military recruits are likely to be young, fit individuals who engage in regular, intense physical activities, the findings from this study should not be considered to reflect the entire spectrum of ECG manifestations in highly trained athletes participating in a variety of sporting disciplines.

Based on the cumulative experience of the authors, on screening competitive athletes there is anecdotal evidence to suggest that patterns similar to the ones present in the African/ Afro-Caribbean athlete's ECG, with convex ST-segment elevation followed by $\mathrm{T}$-wave inversions in leads V1-V4, may be more prevalent in East Asian athletes. These observations need to be confirmed in large-scale studies in competitive athletes.

The echocardiogram of East Asian athletes should also be interpreted with caution since current studies suggest that athletes of Japanese origin exhibit greater LV cavity dilatation in response to exercise compared to both Caucasian and African athletes. $^{36} 37$

\section{Limitations of ethnicity-specific guidelines}

The adoption of ethnicity-specific guidelines for the interpretation of ECG and imaging studies in athletes is hindered by lack of data in several ethnic groups. As with the paradigm of
African/Afro-Caribbean athletes, achievements at the international level usually instigate the need to study in detail expressions of cardiovascular adaptation to exercise. Paradoxically, the endorsement of preparticipation screening by major sporting organisations is likely to stimulate further research in the field in an attempt to differentiate ethnically determined expressions of athlete's heart from quiescent cardiac pathology.

By determining ethnicity, investigators attempt to identify individuals who descend from a similar genetic pool and as such they may share several characteristics. However, physicians evaluating athletic individuals should exercise caution when applying guidelines to specific athletes since the use of the term 'Caucasian' or 'African/Afro-Caribbean' ethnicity does not always ensure a homogeneous population. Results from Italian athletes may not necessarily apply to Northern or Eastern European athletes, and East African marathon runners are anthropometrically distinct from West African sprinters. Furthermore, in the current era where human movement and migration is extensive, determining an athlete's true ethnicity is becoming increasingly challenging. While we applaud the creation and use of ethnic-specific ECG interpretation guidelines, care is warranted in athletes of mixed ethnic origin, where data are even more scarce.

\section{CONCLUSION}

The paradigm of African/Afro-Caribbean athletes illustrates the effect of ethnicity on cardiovascular adaptation to exercise and the importance of adopting ethnicity-specific criteria when evaluating elite athletes. Review of available data indicates that although ethnicity-specific guidelines should be adopted for the interpretation of the 12-lead ECG and echocardiogram of athletes of African/Afro-Caribbean descent, further research is necessary to delineate the limits of normality in a number of other ethnicities.

\section{What this study adds}

- Ethnicity is an important determinant of cardiovascular adaptation to exercise and its impact should be considered when evaluating an athlete.

- African/Afro-Caribbean athletes exhibit marked repolarisation anomalies and significant left ventricular hypertrophy; extrapolation of ECG and echocardiographic criteria used to diagnose potentially serious disease processes in Caucasian athletes would result in an unacceptable number of unnecessary investigations.

- In African/Afro-Caribbean athletes anterior T-wave inversions confined to leads V1-V4, preceded by convex ST-segment elevation, and an absolute maximal left ventricle wall thickness of $16 \mathrm{~mm}$ (13 $\mathrm{mm}$ for female athletes) are likely to represent an ethnically determined, physiological response to exercise. In the absence of cardiac symptoms or a family history of sudden cardiac death or cardiomyopathy the athlete should be allowed to compete.

Acknowledgements The authors are grateful to the charitable organisation Cardiac Risk in the Young (CRY) for providing the portable equipment and support team used for the studies of Caucasian and black athletes in theUK.

Contributors All authors contributed to the writing of the manuscript, reviewed and approved the content. 
Funding MP and SG were funded by research grants from the charitable organisation Cardiac Risk in the Young (CRY). Professor Sanjay Sharma has been a coapplicant on previous grants from CRY to study African/Afro-Caribbean athletes. Studies on French athletes were supported by grants from the Club des Cardiologues du Sport and from the French Ministry of Health and Sport.

\section{Competing interests None.}

Provenance and peer review Not commissioned; externally peer reviewed.

\section{REFERENCES}

1. Sharma S. Athlete's heart—effect of age, sex, ethnicity and sporting discipline. Exp Physiol 2003;88:665-9.

2. Corrado D, Pelliccia A, Heidbuchel $\mathrm{H}$, et al. Recommendations for interpretation of 12-lead electrocardiogram in the athlete. Eur Heart J 2010;31:243-59.

3. Bille K, Fiquiras D, Schamasch P. Sudden cardiac death in athletes. The Lausanne recommendations. Eur J Cardiovasc Prev Rehabil 2006;13:859-75.

4. Rost R. The athlete's heart: historical perspective. In: Maron BJ, ed. Cardiology clinics, the athlete's heart. Philadelphia, PA: WB Saunders Co, 1992:197-207.

5. Maron BJ, Pelliccia A. The heart of trained athletes: cardiac remodelling and the risks of sports, including sudden death. Circulation 2006;114:1633-44.

6. Makan J, Sharma S, Firoozi S, et al. Physiological upper limits of ventricular cavity size in highly trained adolescent athletes. Heart 2005:91:495-9.

7. Pelliccia A, Culasso F, Di Paolo F, et al. Physiologic left ventricular cavity dilatation in elite athletes. Ann Intern Med 1999:130:23-31.

8. Pelliccia A, Maron BJ, De Luca R, et al. Remodeling of left ventricular hypertrophy in elite athletes after long-term deconditioning. Circulation 2002;105:944-9.

9. Pelliccia A, Maron BJ, Di Paolo FM, et al. Prevalence and clinical significance of left atrial remodeling in competitive athletes. J Am Coll Cardiol 2005;46:690-6.

10. Scharhag J, Thünenkötter T, Urhausen A, et al. Echocardiography of the right ventricle in athlete's heart and hearts of normal size compared to magnetic resonance imaging: which measurements should be applied in athletes? Int J Sports Med 2010;31:58-64

11. Pelliccia A, Di Paolo FM, De Blasiis E, et al. Prevalence and clinical significance of aortic root dilation in highly trained competitive athletes. Circulation 2010:122:698-706

12. Pelliccia A, Maron BJ, Spataro A, et al. The upper limit of physiologic cardiac hypertrophy in highly trained elite athletes. N Engl J Med 1991:324:295-301.

13. Sharma S, Maron BJ, Whyte G, et al. Physiologic limits of left ventricular hypertrophy in elite junior athletes: relevance to differential diagnosis of athlete's heart and hypertrophic cardiomyopathy. J Am Coll Cardiol 2002:40:1431-6.

14. Sharma $\mathbf{S}$, Whyte $G$, Elliott $P$, et al. Electrocardiographic changes in 1000 highly trained junior elite athletes. Br J Sports Med 1999;33:319-24.

15. Papadakis M, Basavarajaiah S, Rawlins J, et al. Prevalence and significance of T-wave inversions in predominantly Caucasian adolescent athletes. Eur Heart $J$ 2009;30:1728-35.

16. Wilson MG, Basavarajaiah S, Whyte GP, et al. Efficacy of personal symptom and family history questionnaires when screening for inherited cardiac pathologies: the role of electrocardiography. Br J Sports Med 2008;42:207-11.

17. Pelliccia A, Culasso F, Di Paolo FM, et al. Prevalence of abnormal electrocardiograms in a large, unselected population undergoing pre-participation cardiovascular screening. Eur Heart J 2007:28:2006-10.

18. Pelliccia A, Di Paolo FM, Quattrini FM, et al. Outcomes in athletes with marked ECG repolarisation abnormalities. N Engl J Med 2008;358:152-61.

19. Wasserburger RH. Observations on the juvenile pattern of adult negro males. $A m$ $J$ Med 1955:18:428-37.

20. Xie X, Liu K, Stamler J, et al. Ethnic differences in electrocardiographic left ventricular hypertrophy in young and middle aged employed American men. $A m J$ Cardiol 1994;73:564-7.

21. Shahi M, Foale RA, Poulter NR, et al. Racial differences in cardiac structure and function in essential hypertension. Br Med J 1994:16:1011-14.

22. Basavarajaiah S, Boraita A, Whyte $\mathrm{G}$, et al. Ethnic differences in left ventricular remodelling in highly-trained athletes relevance to differentiating physiologic left ventricular hypertrophy from hypertrophic cardiomyopathy. J Am Coll Cardiol 2008;51:2256-62.

23. Papadakis M, Carre F, Kervio G, et al. The prevalence, distribution, and clinical outcomes of electrocardiographic repolarisation patterns in male athletes of African/ Afro-Caribbean origin. Eur Heart J 2011;32:2304-13.

24. Rawlins J, Carre F, Kervio G, et al. Ethnic differences in physiological cardiac adaptation to intense physical exercise in highly trained female athletes. Circulation 2010;121:1078-85.

25. Di Paolo FM, Schmied C, Zerguini YA, et al. The athlete's heart in adolescent Africans. An electrocardiographic and echocardiographic study. J Am Coll Cardiol 2012;59:1029-36.

26. Choo JK, Abernethy WB III, Hutter AM Jr. Electrocardiographic observations in professional football players. Am J Cardiol 2002;90:198-200.

27. Magalski A, Maron BJ, Main ML, et al. Relation of race to electrocardiographic patterns in elite American football players. J Am Coll Cardiol 2008;51:2250-5.

28. Haissaguerre $\mathbf{M}$, Derval N, Sacher $\mathrm{F}$, et al. Sudden cardiac arrest associated with early repolarisation. N Engl J Med 2008;358:2016-23.

29. Rosso R, Kogan E, Belhassen B, et al. J-point elevation in survivors of primary ventricular fibrillation and matched control subjects: incidence and clinical significance. J Am Coll Cardiol 2008;52:1231-8.

30. Tikkanen JT, Anttonen 0, Junttila MJ, et al. Long-term outcome associated with early repolarisation on electrocardiography. N Engl J Med 2009;361:2529-37.

31. Crouse SF, Meade T, Hansen BE, et al. Electrocardiograms of collegiate football athletes. Clin Cardiol 2009;32:37-42.

32. Noseworthy PA, Weiner R, Kim J, et al. Early repolarisation pattern in competitive athletes: clinical correlates and the effects of exercise training. Circulation 2011:4:432-40.

33. Dabiran S, Tutunchi P, Tutunchi AS, et al. An echocardiographic study of heart in a group of male adult elite athletes. J Teh Univ Heart Ctr 2008;2:107-12.

34. Wilson MG, Chatard JC, Carre F, et al. Prevalence of electrocardiographic abnormalities in West-Asian and African male athletes. $\mathrm{Br} J$ Sports Med 2012; 46:341-47

35. Sun B, Ma JZ, Yong YH, et al. The upper limit of physiological cardiac hypertrophy in elite male and female athletes in China. Eur J Appl Physiol 2007;101:457-63.

36. Nagashima J, Musha $\mathrm{H}$, Takada $\mathrm{H}$, et al. Left ventricular chamber size predicts the race time of Japanese participants in a $100 \mathrm{~km}$ ultramarathon. Br J Sports Med 2006; 40:331-3

37. Kervio G, Pelliccia A, Nagashima J, et al. Alterations in echocardiographic and electrocardiographic features in Japanese professional soccer players: comparison to African-Caucasian ethnicities. Eur J Prev Cardiol. Published online 1 May 2012, doi:10.1177/2047487312447905

38. $\mathbf{N g ~ C T}$, Ong HY, Cheok C, et al. Prevalence of electrocardiographic abnormalities in an unselected young male multi-ethnic South-East Asian population undergoing pre-participation cardiovascular screening: results of the Singapore armed forces electrocardiogram and echocardiogram screening protocol. Europace 2012;14:1018-24.

39. Maron BJ, Thompson PD, Ackerman MJ, et al. Recommendations and considerations related to preparticipation screening for cardiovascular abnormalities in competitive athletes: 2007 update. Circulation 2007;115:1643-55.

40. Corrado D, Pelliccia A, Bjørnstad HH, et al. Cardiovascular pre-participation screening of young competitive athletes for prevention of sudden death: proposal for a common European protocol: consensus statement of the Study Group of Sport Cardiology of the Working Group of Cardiac Rehabilitation and Exercise Physiology and the Working Group of Myocardial and Pericardial Diseases of the European Society of Cardiology. Eur Heart J 2005;26:516-20.

41. Papadakis M, Sharma S. Electrocardiographic screening in athletes: the time is now for universal screening. Br J Sports Med 2009;43:663-8.

42. Wilson MG, Hamilton B, Sandridge AL, et al. Differences in markers of cardiovascular disease between professional football players of West-Asian and Black African descent. J Sci Med Sport 2012;15:266-71.

43. Maron BJ, Doerer JJ, Haas TS, et al. Sudden deaths in young competitive athletes. Analysis of 1866 deaths in the United States, 1980-2006. Circulation 2009:119:1085-92. 